\title{
Improving venous thromboembolism (VTE) prophylaxis in acute urological admissions during out of hours through the introduction of a urological admission proforma
}

\author{
Efstratios Gerakopoulos \\ Leighton Hospital, Mid Cheshire Foundation Trust, UK
}

\begin{abstract}
Venous thromboembolism (VTE) kills more people than breast cancer, road traffic accidents, and AIDS combined, accounting for approximately 25,000 in-hospital deaths in England annually. The cost to the NHS is estimated at $£ 640$ million/annum. The most important element of VTE risk assessment strategy in England is to risk assess all patients for VTE on admission.
\end{abstract}

The aim of our quality improvement programme (QIP) was to monitor our practice regarding VTE prophylaxis of the patients' admitted urgently in our department, and then implement a measure to increase compliance if found to be poor. Our standards were based on the National Institute for Health and Care Excellence (NICE) guidelines which state that all urgently admitted patients must have a completed VTE assessment form within 24 hours of admission and receive appropriate VTE prophylaxis including low molecular weight heparin (LMWH) and/or TED stockings.

Our initial audit was conducted over a period of five weeks. All adult patients acutely admitted out of hours (5pm to 8am) were included. We then introduced a specially designed urological admissions proforma and organised several teaching sessions for junior doctors who facilitated acute admissions. Re-audit was performed using the same methods and timescale measuring improvement. Second re-audit six months after the introduction of the proforma, following the induction of the new cohort of junior doctors. - Primary audit: $n=44$. Proportion of: completed VTE form $=56 \%$, LMWH appropriately prescribed $=65 \%$, TEDS=35\%. VTE related
complications $=3$

- 1st re-audit: $n=42$. Proportion of: completed VTE form=93\%, LMWH appropriately prescribed=83\%,TEDS=64\%. VTE related complications $=0$ - 2nd re-audit:n=43. Proportion of: completed VTE form=92\%, LMWH prescribed $=84 \%$, TEDS=76\%. VTE related complications $=1$

There has been a significant increase of compliance with the NICE guidelines regarding VTE prophylaxis within our department through introducing the specially designed urological admissions proforma and delivering teaching sessions for junior doctors. The implementation of the proforma also led to decreased prevalence of VTE related complications and their subsequent morbidity and mortality.

\section{Problem}

In our healthcare trust there is specialty cross-cover between urology and general surgery during the on-call out-of-hours duties. Consequently the acute urological admissions are being facilitated by either the urology or the general surgery senior house officers, and the clerking of the new patients (including the VTE assessment and prophylaxis) is usually performed by junior doctors who do not regularly work in our urological department.

During our everyday clinical practice, we identified great variability regarding the VTE risk assessment and the appropriate VTE prophylaxis of these patients that were admitted urgently during the cross-cover on calls. That resulted in elderly patients with multiple comorbidities, including urological malignancies and history of previous VTE, to be admitted on our ward without having their VTE assessment form completed or appropriate VTE prophylaxis prescribed, exposing them to increased risk of VTE related complications. That was deemed to be extremely dangerous and our attention was then focused on identifying ways of initially measuring and then improving our performance.

\section{Background}

Venous thromboembolism (VTE), defined as deep vein thrombosis, pulmonary embolism, or both, kills more people than breast cancer, road traffic accidents, and AIDS combined. It accounts for approximately 25,000 in-hospital deaths in England annually[1] and is a cause of considerable morbidity and mortality.[2] The cost to the NHS is estimated at $£ 640$ million per year. It is a disorder that can occur in all races and ethnicities, all age groups, and both genders.[3] Many of the risk factors are well known, such as advanced age, immobility, surgery, and obesity. 
Recently there has been a marked increase in VTE related complications despite national efforts to raise awareness and acknowledge of the need for VTE prevention.[4] The most important element of VTE risk assessment strategy in England is to risk assess all patients on admission. The National Institute for Health and Care Excellence (NICE) has published extensive guidance and formulated a pathway that every clinician must follow in order to reduce the risk of VTE in hospital patients.[5]

\section{Baseline measurement}

Our initial audit was conducted over a period of five weeks (October/November 2013) using a specially designed data collection proforma. All adult patients urgently admitted out of hours (5pm to $8 \mathrm{am})$ under urology were included with no exclusion criteria. The gold standards against which our measurement was made were based on the NICE guidelines which state that:

1. All patients need to have a VTE risk assessment form completed within 24 hours of their admission

2. After their risk assessment all patients should receive the appropriate VTE prophylaxis consisting of subcutaneous low molecular weight heparin (LMWH) or TED stockings, or a combination of the above. Secondary data such as patients' demographics, designation of the person admitting the patient, and any VTE related complications were also recorded during our initial measurement.

The results showed a total number of 44 patients admitted during this period. Mean age was 52.6 (18-92) SD 21.68. Male/Female= 28/16. Designation of person admitting: urology $\mathrm{SHO}=15(34 \%)$, surgical $\mathrm{SHO}=29(66 \%)$. Completed VTE forms on 23 admissions (52\%), low molecular weight heparin (LMWH) appropriately prescribed to 27 patients (61\%), TED stockings applied on 15 patients (34\%).

There were three VTE related complications recorded. One of them was an isolated left lower limb DVT in a 67 years old female, and the other two presented in a 72 years old male patient that suffered from known urological malignancy, with a left lower limb DVT that led to a minor PE. Further statistical analysis was conducted by splitting the data into two groups:

Group A: Admissions undertaken by urology SHOs: completed VTE forms $=86 \%$, LMWH prescribed $=86 \%$, TEDS applied $=73 \%$. Group B: Admissions undertaken by surgical SHOs: completed VTE forms $=35 \%$, LMWH prescribed $=42 \%$, TEDS applied $=11 \%$.

Table 1 shows the variance in clinical practice between the urology and the surgical SHOs.

See supplementary file: ds4703.doc - "table 1"

\section{Design}

After careful analysis of the primary results, our performance was deemed to be poor and we recognised the need for improvement in our practice. Several informal meetings were held within our department involving a wide range of healthcare professionals, including junior doctors, registrars, consultants, advanced nurse practitioners, and staff nurses from the ward. During these meetings there was constructive exchange of ideas and previous experiences from each of the members of our urological department and some very important conclusions were drawn.

It was obvious that junior doctors, especially the general surgery senior house officers (SHO) were not aware of the necessity for VTE prophylaxis in all acute urological admissions without any exclusion. Another problem was the inavailability of the VTE form in clinical areas of our hospital.

There was a clear need for intervention, and the best way to accomplish that was to design an acute urological admissions proforma and educate the junior doctors on how to use it. This proforma included the VTE assessment form in it as well as many other important clinical information regarding the patient who was being urgently admitted.

\section{Strategy}

A team consisting of a senior house officer, a senior registrar, a consultant, and an advanced nurse practitioner was given the responsibility to design the acute urological admissions proforma (AUAP). The structure of the AUAP was based on already existing proformas that were used by other specialties in our hospital. Great care was taken in order to include all the crucial clinical information specifically aimed at acute urological clinical conditions. One of the pages of the AUAP was the VTE assessment form that is necessary to be completed for each patient admitted. That made it easy for the junior doctors to allocate and complete it and then prescribe the appropriate VTE prophylaxis.

After designing the proforma it was distributed to all the consultants in our department and every suggestion for its improvement was taken into account and the structure of the AUAP was adjusted. Following that, a pilot study was designed and conducted and the AUAP was used for a trial period of one month. Feedback from junior doctors who used the proforma during the trial was collected and evaluated and the final improving cycle of our project was completed. Teaching sessions were then organised by the senior registrars of our department in order to disseminate the results of our initial audit to the junior doctors working in both the general surgical and the urological firms. During these sessions the need for improvement was stressed and instructions were given about how the proforma should be used. In order to reduce the Hawthorne effect, our team did not disseminate the timeframe of when our post implementation measurements were going to take place in order to prevent any false positive improvement in the juniors' performance. The AUAP was finally disseminated to accident and emergency department, the acute surgical assessment unit, and all the surgical wards.

\section{Results}


Following the official introduction of the proforma re-audit was conducted using the same standards and timescale as for the primary audit (February/March 2014). The results showed that there were 42 patients admitted urgently in our department. Mean age was 48.48 (17 to 88) SD 22.5. Male/female= 30/12. Designation of person admitting: urology $\mathrm{SHO}=15(36 \%)$, surgical $\mathrm{SHO}=27$ (64\%). Completed VTE forms on 39 admissions (93\%), low molecular weight heparin (LMWH) appropriately prescribed to 35 patients (83\%), TED stockings applied on 27 patients (64\%). There were no VTE related complications identified. Further statistical analysis was again conducted by splitting the data into two groups:

Group A: Admissions undertaken by urology SHOs: proportion of completed VTE forms on admission $=100 \%$, LMWH prescribed= $93 \%$, TEDS applied $=87 \%$. Group B: Admissions undertaken by surgical SHOs: proportion of completed VTE forms on admission= $92 \%$, LMWH prescribed $=78 \%$, TEDS applied $=60 \%$.

After the encouraging results of our re-audit measurement, the AUAP became an official departmental policy and its use became obligatory for all the junior doctors who were facilitating acute urological admissions. Following a period of six months (shortly after the induction of the new cohort of junior doctors), a third and final audit was conducted using the same methods, standards, and timescale (August/September 2014). The final results identified 43 patients admitted urgently in our department. Mean age was 53.43 (17-92) SD 22.8. Male/female=28/15. Designation of person admitting: urology $\mathrm{SHO}=18(42 \%)$, surgical $\mathrm{SHO}=25(58 \%)$. Completed VTE forms on 39 admissions (92\%), low molecular weight heparin (LMWH) appropriately prescribed to 36 patients (84\%), TED stockings applied on 33 patients (76\%). There was one VTE related complication recorded, which was specifically a right lower limb DVT in a 77 years old male patient. Final statistical analysis was again conducted by splitting the data into two groups:

Group A: Admissions undertaken by urology SHOs: proportion of completed VTE forms on admission $=100 \%$, LMWH prescribed $=$ $97 \%$, TEDS applied $=93 \%$. Group B: Admissions undertaken by surgical SHOs: proportion of completed VTE forms on admission= $93 \%$, LMWH prescribed $=91 \%$, TEDS applied $=88 \%$.

Table 2 shows in summary the improvement that was achieved by using the AUAP during the three different audits.

See supplementary file: ds 4701 .doc - "table 2"

\section{Lessons and limitations}

The most important lesson that we have learned through completing this project was that improvement in our everyday clinical practice is easier and more simple that us junior doctors think. All it takes is effective and creative teamwork along with healthcare professionals who are motivated in improving the care they deliver to patients. A multidisciplinary team approach to common clinical problems, such as VTE prophylaxis in our case, is the way forward in our opinion. Every team member brings in its own special attributes and abilities that must be valued and used appropriately for a quality improvement project to be successful.
Hesitation was the main problem that we faced during conducting our project. Hesitation from the side of the junior doctors to propose something new, hesitation from the side of the consultants to accept that change in practice was needed, and finally hesitation form the side of other healthcare professionals to actively participate. Once these issues were solved, our project was successfully completed and brought great improvement as our results show. We strongly believe that the AUAP we introduced is a cost effective way of improving VTE prophylaxis in the long term, and can prevent devastating VTE related complications and their subsequent morbidity and mortality.

The small number of patients that were recorded during our three audits is the only limitation of our project and stresses the need for further similar measurements on an extended timescale that can include a bigger number of patients and draw further conclusions.

\section{Conclusion}

There has been a significant increase of compliance with the NICE guidelines regarding VTE prophylaxis within our department through the introduction of the specially designed acute urological admissions proforma (AUAP).

1. The proportion of completed VTE forms within 24 hours of admission increased to $92 \%$ from $52 \%$

2. The proportion of patients receiving appropriate LMWH increased to $84 \%$ from $61 \%$

3. The proportion of patients having TEDS fitted increased to $76 \%$ from $34 \%$

Statistical analysis revealed that the implementation of the proforma led to decreased prevalence of VTE related complications, avoiding subsequent morbidity and mortality. It also increased the level of care provided as well as the overall satisfaction and positive experience of our patients, minimising the risk of possible complaints and litigation. The reduction in the VTE related complications has also resulted in reduced costs for our department, an aspect of patients care that is becoming increasingly important in modern practice. We strongly believe that in addition to the introduction of the proforma, the formal teaching of the juniors doctors regarding the necessity for thorough VTE risk assessment and appropriate prophylaxis for the acute urological patients is equally as important.

\section{References}

1. Carrier M, Le Gal G, Wells PS, Rodger MA. Systematic review: case-fatality rates of recurrent venous thromboembolism and major bleeding events among patients treated for venous thromboembolism. Ann Intern Med 2010;152(9):578-89.

2. Heit JA, Silverstein MD, Mohr DN, Petterson TM, O'Fallon WM, Melton LJ III. Predictors of survival after deep vein thrombosis and pulmonary embolism: a population-based, cohort study. Arch Intern Med 1999;159(5):445-53.

3. Pengo V, Lensing AW, Prins $\mathrm{MH}$, et al; Thromboembolic 
Pulmonary Hypertension Study Group. Incidence of chronic thromboembolic pulmonary hypertension after pulmonary embolism. N Engl J Med 2004;350(22):2257-64.

4. Tagalakis V, Patenaude V, Kahn SR, Suissa S. Incidence of and mortality from venous thromboembolism in a realworld population: the Q-VTE Study Cohort. Am J Med 2013;126(9):e13-e21.

5. Reducing the risk of venous thromboembolism in hospital patients. Available at www.nice.org.uk

\section{Declaration of interests}

Nothing to declare.

\section{Acknowledgements}

Mr Snehal Patel, Ann Baker, Mr Rono Mukherjee. 